\title{
CARDINAL ADDITION AND THE AXIOM OF CHOICE
}

\author{
BY J. D. HALPERN AND PAUL E. HOWARD
}

Communicated by F. W. Gehring, October 10, 1973

Using the axiom of choice (AC) one can prove that cardinal arithmetic is trivial for infinite cardinals. That is:

$$
m+n=\sup (m, n)=m \cdot n \quad \text { if one of } m, n \text { are infinite. }
$$

These two laws follow directly from the simpler looking laws:

(1) $m+m=m$ for all infinite cardinals $m$, and

(2) $m \cdot m=m$ for all infinite cardinals $m$.

In 1924 Tarski [2] gave a proof of (AC) from (2) and asked [3] if (AC) is provable from (1). We give a negative answer to this question by exhibiting a permutation model of ZFU (Zermelo-Fraenkel set theory modified so as to allow urelements) in which $\mathrm{C}_{2}$ (the axiom of choice for families whose elements are pairs) is false but (1) is true. ${ }^{1}$

A permutation model (Specker [1]) is determined by a set of points ${ }^{2}$ (i.e. urelements) $U$, a group $G$ of permutations of $U$, and a conjugated filter $J$ of subgroups of $G$.

Let $U$ be a countable set of points. $G$ and $J$ will be described in terms of a 1-1 correspondence between $U$ and $\omega^{(\omega)}=\left\{s: \omega \rightarrow \omega \mid(\exists n)(\forall j>n) s_{j}=0\right\}$. For notational simplicity, in the sequel we will identify $U$ with $\omega^{(\omega)}$. For $s \in U$, let the pseudo length of $s=\mu k\left(\forall n>k, s_{n}=0\right)$. Call a set $A \subseteq U$ bounded if there is a finite bound on the pseudo lengths of elements of $A$. For any permutation $\varphi$ of $U$ call $\{a \mid \varphi(a) \neq a\}$ the support of $\varphi$. Let $G=\{\varphi \mid \varphi$ is a permutation of $U$ and the support of $\varphi$ is bounded $\}$. For

AMS (MOS) subject classifications (1970). Primary $02 \mathrm{~K} 05$.

1 The proof of our result was completed near the end of December 1972. We learned subsequently that G. Sageev (Notices Amer. Math. Soc. 20 (1973), A22) had already shown that the answer to Tarski's question was negative even in the context of set theory with regularity.

2 Permutation models can also be used for set theories whose axioms are those of ZF except that the axiom of regularity is weakened or eliminated. Specker deals with such a theory but his development of permutation models carries over verbatim for ZFU. We use ZFU rather than such a theory because it seems more natural. 
$n \in \omega, s \in U$ let $n \mid s$ (read " $s$ after $n$ ") $=\left(s_{n}, s_{n+1}, \cdots\right)$ and let $A_{s}^{n}=\{t \in U$ : $n|t=n| s\}$ (read "the $n$-block containing $s$ "). Note that $\varphi \in G$ implies that for some $k$, the support of $\varphi$ is a subset of $A_{\mathbf{0}}^{k}$. Also note that $s \in U$ is uniquely determined (for each $n \in \omega$ ) by $s \mid n$ and $n \mid s$. We will call $s \mid n$ the $n$-location of $s$. For each $n \in \omega$ let $G_{n}=\{\varphi \in G: 1$ and 2 and 3$\}$ where

1. $\varphi$ fixes the $n$-block containing 0 pointwise. ( 0 is the sequence which is identically 0 .)

2. $\varphi$ fixes $\left\{A_{s}^{n}: s \in U\right\}$, i.e., $n|s=n| t$ implies $n|\varphi(s)=n| \varphi(t)$.

3. $\varphi$ preserves $n$-locations, i.e., $s|n=\varphi(s)| n$. Note that $G_{0}=\{\varphi \in G$ : $\varphi(\mathbf{0})=\mathbf{0}\}$. Also note that if $f$ is a 1-1 partial function on $U$ into $U$ with bounded domain and satisfying 1, 2 and 3 then $f$ has an extension in $G_{n}$. Let $J=$ the filter of subgroups generated by the $G_{n}, n \in \omega$.

Briefly the description of the model $M$ is as follows: Let $R$ be the function on ordinals defined recursively by $R(0)=U$ and $R(\alpha)=P\left(\bigcup_{\beta<\alpha} R_{\beta}\right)$ for $\alpha>0$. Then $V=\bigcup_{\alpha \in \text { ord }} R(\alpha)$ is called the set universe over $U$. Any permutation $\varphi$ of $U$ extends uniquely to an automorphism (with respect to $\in) \varphi^{*}$ of $V$. We will confuse $\varphi^{*}$ with $\varphi$ in the future. Call an $x \in V$, $J$-symmetric if there exists $H \in J$ such that $H$ fixes $x$, i.e., $\varphi \in H \rightarrow \varphi(x)=x$. Then $M$ is just the substructure of $V$ consisting of all those sets $x$ such that $x$ and every element of the transitive closure of $x$ is $J$-symmetric.

THEOREM 1. $C_{2}$ is false in $M$.

Proof. The set of all (unordered) pairs of elements of $U$ is in $M$. However for any $k$ there is $\varphi \in G_{k}$ such that $\varphi$ interchanges two elements of $U$. Hence there is no $J$-symmetric choice set for this set of pairs.

It remains to show that (1) holds in the structure $M$. To this end consider a set $y \in M$, and take $k_{0}>0$ such that $G_{k_{0}-1}$ fixes $y$ and let:

$y_{1}=\left\{x \in y: G_{k_{0}}\right.$ fixes $\left.x\right\}$,

$y_{2}=\left\{x \in y: G_{k_{0}}\right.$ does not fix $x$ and there exists a bounded set $A \subseteq U$ such that $\forall \varphi \in G_{k_{0}}(\varphi$ fixes $A$ pointwise implies $\varphi(x)=x)$ \},

$y_{3}=\left\{x \in y:\right.$ For all bounded sets $A \subseteq U \exists \varphi \in G_{l_{0}}(\varphi$ fixes $A$ pointwise and $\varphi(x) \neq x)\}$.

Then $y$ is the disjoint union of $y_{1}, y_{2}, y_{3}$. Furthermore $y_{1}, y_{2}, y_{3} \in M$ since $G_{k_{0}}$ fixes each of them. (Actually $G_{k_{0}-1}$ fixes $y_{3}$.) $y_{1}$ is well-orderable in $M$ since $G_{k_{0}}$ fixes each element and hence a well-ordering of $y_{1}$. We will show that for $z=y_{2}$ or $z=y_{3}$ there is a 1-1 function $F$ with domain $2 \times z$ and range $z$ such that $G_{k_{0}+1}$ fixes $F$ and hence $F \in M$. This shows that every cardinal in $M$ is the sum of three cardinals one of which is well-orderable and the other two satisfy the equation $2 m=m$ and hence that (1) is true in the structure $M$. (Note. If $y$ is finite, it follows that $y_{2}$ and $y_{3}$ must be empty.) 
We are able to construct our function $F$ for $y_{2}$ from two functions

such that

$$
f_{0}, f_{1}: U-A_{0}^{k_{0}} \stackrel{1-1}{\stackrel{1-1}{\longrightarrow}} U-A_{0}^{k_{0}}
$$

(a) $f_{0}, f_{1}$ have disjoint ranges,

(b) $f_{0}, f_{1}$ are each fixed by $G_{k_{0}+1}$ and thus $f_{0}, f_{1} \in M$,

(c) $f_{0}, f_{1}$ preserve $k_{0}$-locations and each maps $k_{0}$-blocks to $k_{0}$-blocks; so the restrictions of $f_{0}, f_{1}$ to a bounded set have extensions in $G_{k_{0}}$.

A crucial lemma in this construction is the following:

LEMMA. Let $x \in y_{2}$. If $A$ and $A^{\prime}$ are bounded subsets of $U$ such that $\forall \psi \in G_{k_{0}}$ ( $\psi$ fixes $A$ or $A^{\prime}$ pointwise implies $\psi(x)=x$ ), if $\varphi, \varphi^{\prime} \in G_{k_{0}}$, $\varphi \supseteq f_{0}\left|A, \varphi^{\prime} \supseteq f_{0}\right| A^{\prime}$ then $\varphi(x)=\varphi^{\prime}(x)$. (Similarly for $f_{1}$.)

In the case of $y_{3}$ we show that for every $x \in y_{3}$ there is an infinite set $D_{x} \in M$ such that

(1) $D_{x} \subseteq y_{3}$,

(2) $\forall \varphi \in G_{k_{0}+1}, \forall z_{1}, z_{2} \in D_{x}\left(\varphi\left(z_{1}\right)=z_{1}\right.$ iff $\left.\varphi\left(z_{2}\right)=z_{2}\right)$ and $\left(z_{1} \neq z_{2} \rightarrow\right.$ $\left.\varphi\left(z_{1}\right) \neq z_{2}\right)$,

(3) $x \in D_{x}$.

It then follows from general considerations concerning permutation models that there exists in $M$ a decomposition of $y_{3}$ into simultaneously denumerable sets. The desired function $F$ can be defined in terms of this decomposition.

Details will appear in a forthcoming paper.

\section{REFERENCES}

1. Ernst Specker, Zur Axiomatik der Mengenlehre (Fundierungs und Auswahlaxiom), Z. Math. Logik Grundlagen Math. 3 (1957), 173-210. MR 20 \#5738.

2. Alfred Tajtelbaum-Tarski, Sur quelques theoremes qui equivalent a l'axiome du choix, Fund. Math. 5 (1924), 147-154.

3. ——, Probleme 31, Ibid., 338.

Department of Mathematics, University of Toledo, Toledo, OHio 43606

Department of Mathematics, Eastern Michigan University, Ypsilanti, MichIGAN 48197

Current address (J. D. Halpern): Department of Mathematics, University of Alabama in Birmingham, Birmingham, Alabama 35294 